\title{
Upgrading the ICRF data acquisition system at ASDEX Upgrade
}

\author{
H. Faugel ${ }^{\mathrm{a}}$, V. Bobkov ${ }^{\mathrm{a}}$, H. Eixenberger ${ }^{\mathrm{a}}$, Y. Podoba $^{\mathrm{a}}$, I. Stepanov ${ }^{\mathrm{a}, \mathrm{b}}$, ASDEX Upgrade Team $^{\mathrm{a}}$ \\ ${ }^{a}$ Max-Planck-Institute for Plasma Physics, EURATOM Association, 85748 Garching, Germany \\ ${ }^{b}$ Department of Applied Physics, Gent University, 9000 Gent, Belgium
}

\begin{abstract}
The ICRF system at ASDEX Upgrade has proved to be very flexible in use, from high power heating down to moderate or low power levels needed for vessel conditioning. As a consequence of these wide varieties of applications the present capabilities of the data acquisition system became a limiting factor, as the CAMAC system could only record up to about 500 ksamples per channel with 12 bit resolution. The upgrade to the SIO (serial I/O data acquisition) system allows a constant sampling time of $5 \mu$ s during the whole plasma discharge with an increased resolution of 16 bit. The SIO system was already used in the last campaign of ASDEX Upgrade with 96 channels and will be further expanded. At low power ICRF experiments the output voltage of the linear rf-detectors was rather low, which led to large measurement errors. As a consequence of this the linear detectors were replaced by logarithmic detectors, expanding the dynamic range from $30 \mathrm{db}$ to $60 \mathrm{~dB}$. Each of the new rf-detectors has two rf inputs and allows to measure the phase difference between the inputs.
\end{abstract}

Keywords: ASDEX Upgrade, ICRF, ICRH, Data Acquisition, rf-detector PACS: 52.50.Qt, 07.05.Hd, 07.57.Kp

\section{INTRODUCTION}

The ICRF system at the ASDEX Upgrade tokamak is in operation since 1992. The system consists of four rfgenerators, a complex transmission and matching system and four ICRF-antennas in the vessel. A maximum rfpower of 7.2 MW was achived; up to $5.5 \mathrm{MW}$ is applied for experiments regularly [1].

In order to save time and money the ICRF's CAMAC data acquisitions system of the ASDEX tokamak was reused in the startup phase in the early 1990s. As it became necessary to replace the CAMAC systems the CODAC group of ASDEX Upgrade started to implement the SIO (Serial I/O) data acquisition system in 2009. This DAQ (data acquisition) system uses the PCIexpress (Peripheral Component Interconnect express) interface of a host computer. Further requests were to replace linear rf-detectors with logarithmic rf-detectors to increase the dynamic range of measurements. The new designed rf-detector also allows a phase measurement between two signals, allowing a better characterization of the antennas.

\section{LIMITATIONS OF THE CAMAC DATA ACQUISITION}

During 20 years of operation the CAMAC DAQ system proved to be a reliable system that can handle a large number of channels. Before we began to outsource parts of the CAMAC system to other DAQ systems, this peaked in about 250 channels distributed in five CAMAC crates filling up two 19-inch racks. As the CAMAC standard (EUR 4100) was originally defined in 1972 by ESONE Committee and ESONE was hibernated in 2001 [2], it became clear that the CAMAC system has reached the end of its lifetime. Also using the CAMAC DAQ showed increasingly limits, e.g. a time resolution of only $20 \mu$ s during long plasma discharges, caused by the storage depth of the system, resulted in a coarse coverage of fast plasma induced changes like ELMs (edge localized mode). During experiments with low power, like wall conditioning [3], the input voltage range of $+/-5 \mathrm{~V}$ with a resolution of 12 bit (approx. $2.44 \mathrm{mV}$ per digit) was limiting, as well as the dynamic range of the used rf-detectors which was about $30 \mathrm{~dB}$.

\section{OUTSOURCING GENERATOR SIGNALS FROM CAMAC}

A large block of the ICRF DAQ are the signals from the four rf-generators. These signals were sampled to allow an analysis of generator shut offs during plasma shots and as an information source of the general generator 
conditions e.g. indicating the need to retuned a generator to achieve maximum output power. Up to 16 channels per generator were used, which was about $1 / 4$ of the CAMAC DAQ channels. All these generator related signals have been moved to a 96 channel National Instruments LabView DAQ system, which also works as a stand alone system, e.g. for maintenance, service or tuning operations of generators. With an input voltage range of +/- $10 \mathrm{~V}$ the ADCs are perfectly covering the $0-10 \mathrm{~V}$ signal level of the rf-generators. The signals recorded are: voltages and currents of the anode power supplies of the tetrodes, control and screen grid currents, forward and reflected power at the driver and final stage. The plate dissipation of the tetrodes is calculated using the DC power input parameters of the stages where the netto output power (forward - reflected) is subtracted.

\section{SIO (SERIAL INPUT/OUTPUT) DATA AQUISITION}

The SIO DAQ concept (Fig. 1) was developed to provide a simple and versatile method for connecting external measuring systems to computers via standard optical links. Currently the connection to the host computer is maintained by a PCIexpress card, called SIO card, which provides four bidirectional optical HOTLink II data ports and an optical central timer clock port. Each HOTLink II data port can communicate with a Pipe interface hosted in a $3 \mathrm{U}(133 \mathrm{~mm})$ high 19 -inch subrack, equipped with an active back plane bus allowing the installation of up to 20 ADC cards per subrack. Each ADC card has either 2 or 4 inputs, allowing to access up to 320 ADC channels with a single SIO card when all four optical links are used. For the ICRF data acquisition system a new 4 channel ADC card with 16 bit resolution was developed, the sampling rate of each channel is $200 \mathrm{kHz}$. A SPI (Serial Peripheral Interface) bus on the ADC card is used to set the amplification of the input signal, turning on and off an anti-aliasing filter and allows offset compensation by adding a voltage via a digital/analog converter. All these settings are part of the shot file header, which contains the settings for the DAQ system for the current shot. A 1-wire bus allows reading each ADC card configuration. The optical link between SIO card and the Pipe interface provides galvanic isolation. The starting time of the data acquisition is set by time mark which the host computer receives from the central DAQ computer of ASDEX Upgrade. This time mark is written to the SIO card and is compared with the central timer clock input. When both time marks are equal, the data acquisition starts: the SIO card sends a given number of sample requests to the Pipe interface. For each sample request to the Pipe interface a time stamp is recorded, after finishing a requested data acquisition the data from each ADCs is serially shifted over a 16 bit wide active back plane bus to a CPLD (complex programmable logic device) in the Pipe interface which does the parallel/serial conversion and transmits the data via a HOTLink II interface to the SIO card. The SIO card transfers the received data with DMA (direct memory access) in the RAM of the host computer. The maximum transfer rate of each HOTLink II interface is $500 \mathrm{Mbit} / \mathrm{s}$. Theoretically, a fully equipped 19-inch subrack with 80 16-bit ADCs allows a maximum sample frequency of about $300 \mathrm{kHz}$ per cannel. To shift this limit the SIO II DAQ system was developed using optical data ports that can transfer up to $2.5 \mathrm{Gbit} / \mathrm{s}$ [4].

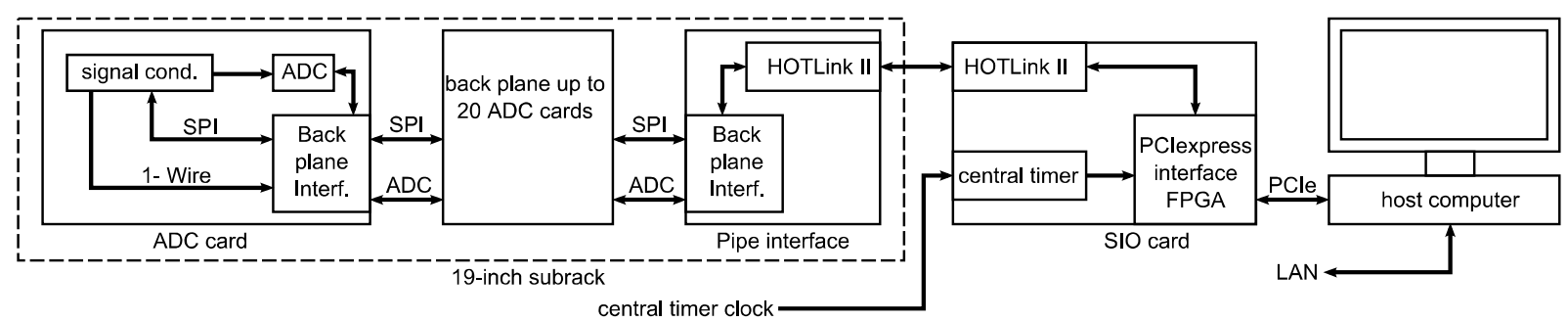

FIGURE 1. Overview of the serial I/O (SIO) data acquisition. The host computer equipped with a PCIexpress interface card, called SIO card that connects via light fibers to the Pipe interface which controls the ADC cards mounted in the 19-inch subrack. Data acquisition is initiated when the timer mark broadcasted from the central timer matches the time mark set via the shot file header and starts to record the programmed number of samples.

\section{REPLACING LINEAR RF-DETECTORS WITH LOGARITHMIC RF-DETECTORS}

ASDEX Upgrade ICRF system uses four high power rf-generators with a maximum output power of 2 MW each. The generators have basically two operation modes, a pulsed (max. $10 \mathrm{~s}$ ) high power mode with a power range from $50 \mathrm{~kW}$ up to $2 \mathrm{MW}$ and a c.w. mode with output power ranging from 1 to $60 \mathrm{~kW}$, giving a ratio of 1:2000 in possible power setting. This high ratio of $33 \mathrm{~dB}$ is not easy to cope using linear detectors, at the low power settings the measurement have been inaccurate, limited by linearity and offset changes of the rf-detector and by the 12 bit 
resolution of the ADC. For high power operation a number of rf-detectors had to be equipped with additional attenuators to prevent saturation. The new developed rf-detectors (Fig. 2) uses a logarithmic detector IC [5] with a dynamic range better than $60 \mathrm{~dB}$ whereas the linear detector had a range of about $30 \mathrm{~dB}$. This detector IC features a true RMS (root mean square) power measurement instead of a peak voltage measurement in the linear rf-detector. Measuring power can reduce errors significantly, e.g. when two antennas operating at different frequencies show cross coupling. Measuring the phase is done by using the input signals splitted with a $0^{\circ}$ and a $90^{\circ}$ power splitter. This allows measuring $0-360^{\circ}$ phase shift between the signals even if the phase detector IC [6] itself can only distinguish $180^{\circ}$. Furthermore the maximum input level has been raised from 18 to $23 \mathrm{dBm}$, which does cancel the necessity of using additional attenuator preventing saturation of the rf-detectors. With an average slope of 50 $\mathrm{mV} / \mathrm{dB}$, the logarithmic rf-detector increased the measurement resolution significantly at low power input. A change of one $\mathrm{dB}$ at the input of the logarithmic rf-detector results in approximately 160 digits at the 16-bit ADC of the SIO system with in range from -40 to $23 \mathrm{dBm}$. To reach this resolution with the 12-bit CAMAC system and the linear detector an input level of $17 \mathrm{dBm}$ was needed, which was close to maximum input level it could handle. The phase measurement capability of the rf-detector is also being used in laser interferometry at ASDEX Upgrade [7].
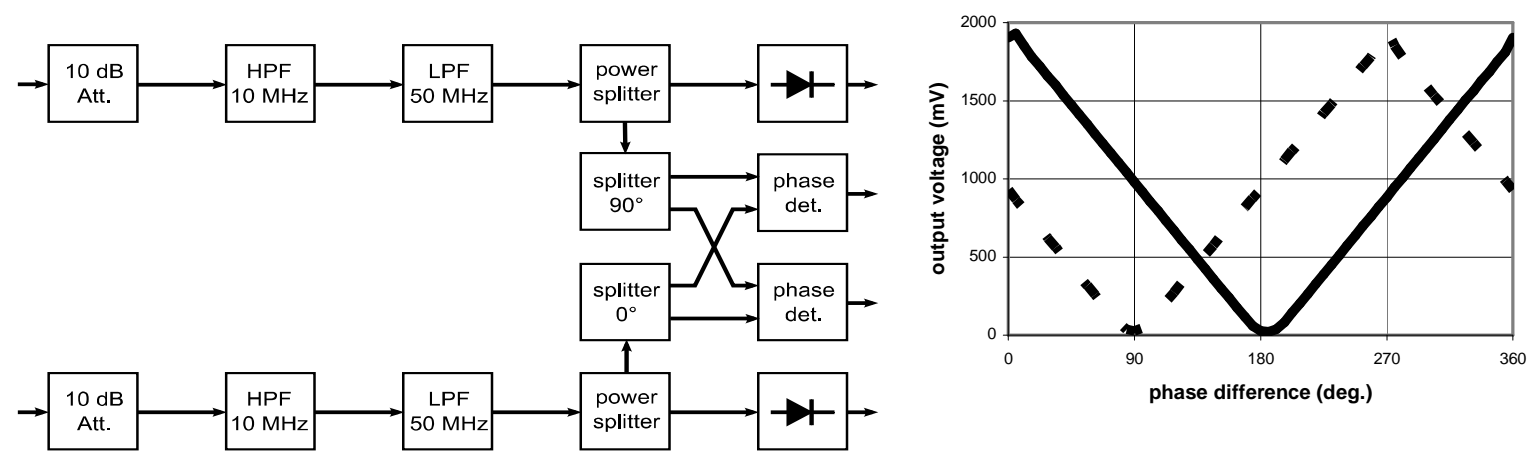

FIGURE 2. The left part shows the simplified schematic of the rf-detector. A $10 \mathrm{~dB}$ attenuator at the input increases the maximum input level before the logarithmic detector saturate and maintains a better VSWR. The $10 \mathrm{MHz}$ high pass filter suppresses noise picked up by the ICRF measurement system in the tokamak hall, the following low pass filter suppresses harmonics of the ICRF frequency. The right graph shows the characteristic of the two phase detector a function of phase difference of the two input signals. Within a range of about 250 to $1600 \mathrm{mV}$ output voltage the signals are linear, allowing precise phase measurements with a resolution of fractions of a degree.
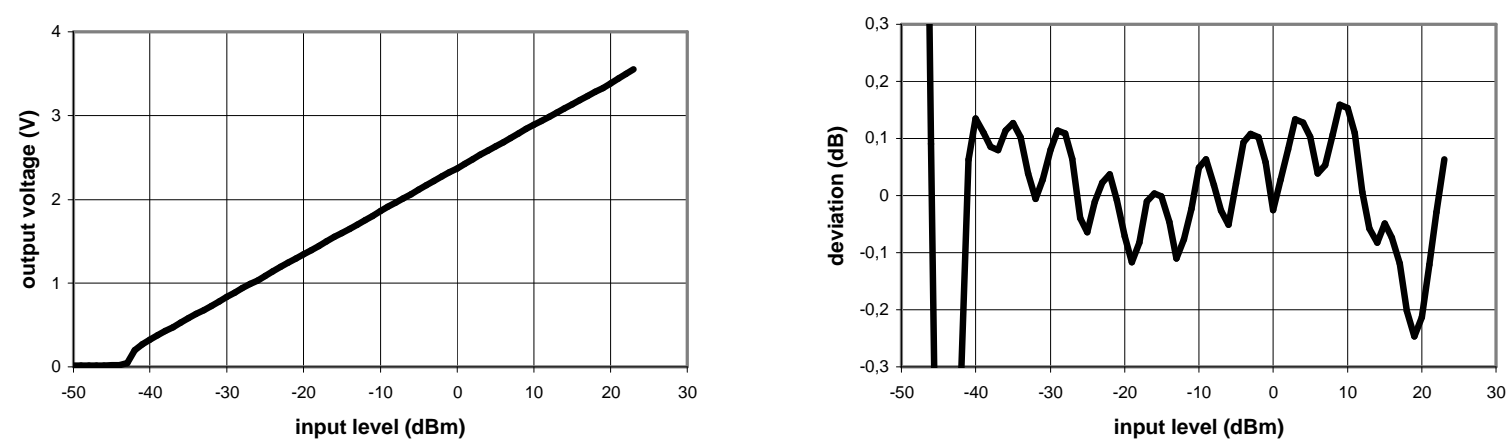

FIGURE 3. The left graph shows the characteristic of the logarithmic rf-detector as function of the input power. Using a linear equation to approximate the behavior of the detector over an input range from -40 to $23 \mathrm{dBm}$ resulted in the right plot showing the deviation from this approximation. To reduce the error even further each detector was calibrated and the data was put in a XML file to be accessible for data evaluation.

\section{DATA INTERPRETATION}

The major drawback of logarithmic detectors is that the output voltage needs a quite complicated procedure to be calculated into physical units. Over an range of -40 to $23 \mathrm{dBm}$ the input power can be linearly approximated within 
an error of +/- $0.25 \mathrm{~dB}$ (Fig. 3), which is sufficient for a number of applications. For a more precise data interpretation each log-rf-detector was calibrated in one dB steps using a DDS (Direct Digital Synthesis) signal generator as classic rf-signal generators showed a significant amount of error in the output level. The calibration data was used to create a XML (Extensible Markup Language) file that contains this information for further processing of raw data, like creating Level 1 shot files where the measured voltages are converted into physical values.

\section{OUTLOOK AND CONCLUSIONS}

After a few months of operations the SIO DAQ has already shown its capabilities, and the number of channels will be increased from 96 to over 200 by the end of 2013. The increased resolution of $0.31 \mathrm{mV} /$ digit instead of 2.44 $\mathrm{mV} /$ digit is not only of theoretical interest (Fig. 4), e.g. the scaling of the phase detector is only $10 \mathrm{mV} / \mathrm{degree}$ and the phase changes between voltage and current probes during a discharge in range of a few degrees. Due to the much higher data throughput, of the SIO DAQ the ICRF shot files are earlier accessible to the ASDEX Upgrade and ICRF session leaders, giving more time for decisions about settings for the next shot. The capability of the new rf-detector to measure the phase between two signals is something which is e.g. essential for characterizing new antennas or matching systems in plasma operation.

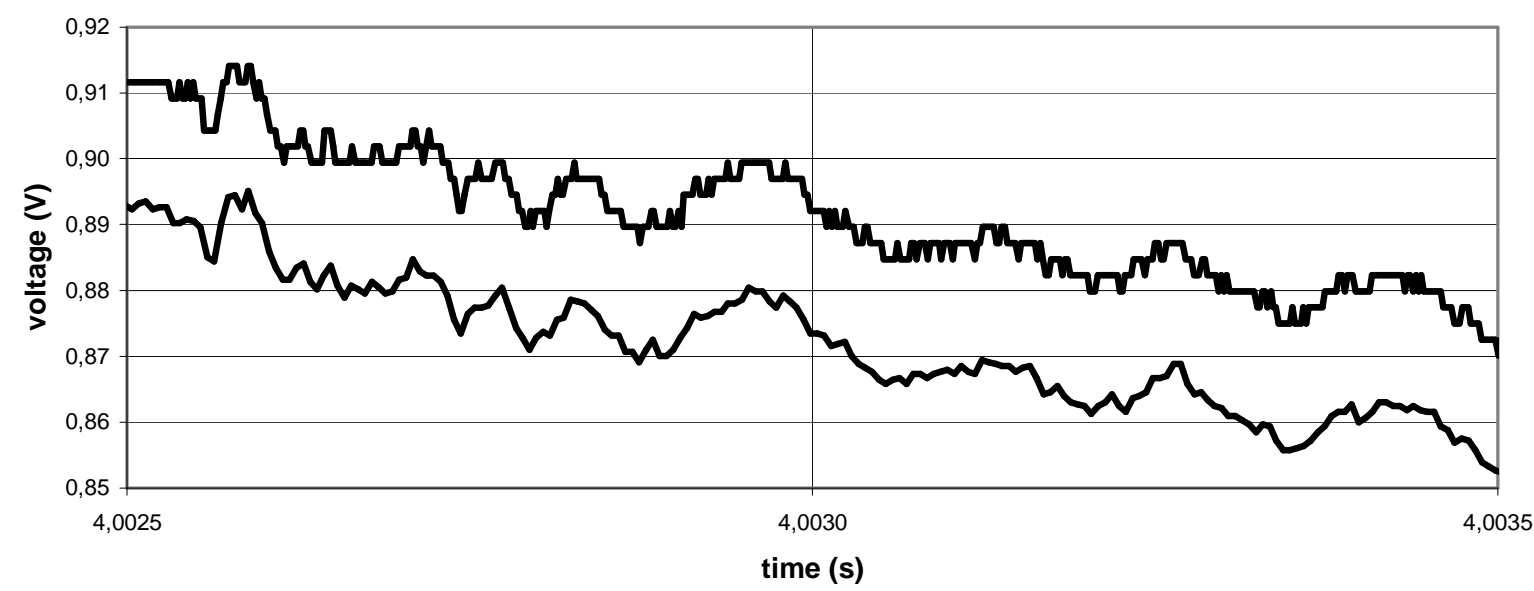

FIGURE 4. Two traces of the same phase signal during ASDEX Upgrade shot \#29828 (standard H-Mode). The upper trace was recorded using the CAMAC DAQ with $+/-5 \mathrm{~V}$ input range and 12 bit resolution, the lower trace was recorded using the SIO DAQ with +/- $10 \mathrm{~V}$ input range and 16 bit resolution and is shifted downward by $20 \mathrm{mV}$ to separate the traces in the plot. It is clearly visible that the signal of the SIO DAQ is of higher quality.

\section{ACKNOWLEDGMENTS}

I would like to thank the ASDEX Upgrade electronics group for their efforts in bringing the SIO DAQ system into operation. Also many thanks to Hans Huber from the ITZ department of IPP for manufacturing the logarithmic detectors and to Bernd Eckert from the ICRF group for calibrating the detectors.

\section{REFERENCES}

1. H. Faugel et al. "The ASDEX Upgrade ICRF system: Operational experience and developments", Fusion Engineering and Design 74, November 2005, pp. 319-324

2. http://esone.web.cern.ch/ESONE/hibernation.pdf

3. A. Lyssoivian et al., "RF physics of ICWC Discharge at High Cyclotron Harmonics", this conference

4. K. Behler et al., "Deployment and future prospects of high performance diagnostics featuring serial I/O (SIO) data acquisition (DAQ) at ASDEX Upgrade", Fusion Engineering and Design 87, 2012, pp. 2145-2151.

5. Datasheet Analog Devices AD 8362, http://www.analog.com/en/rfif-components/detectors/ad8362/products/product.html

6. Datasheet Analog Devices AD 8302, http://www.analog.com/en/rfif-components/detectors/ad8302/products/product.html

7. A. Mlynek et al., "Improved phase detection schemes for plasma interferometry", to be published EPS 2013 